\title{
Quarter-sweep arithmetic mean algorithm for water quality model
}

\begin{abstract}
In the previous studies, approximate solutions of water quality model were calculated by Half-Sweep Arithmetic Mean (HSAM) and Half-Sweep Geometric Mean (HSGM) methods using the HSCN finite difference scheme. Based on the $\mathrm{CN}$ finite difference scheme, however, we investigate application of the Quarter-Sweep Arithmetic Mean (QSAM) method in assessing the water quality model. In this paper, the formulation of the FSAM, HSAM and QSAM needs will be presented. Finally, results on numerical experiments conducted validate that the QSAM method has been shown to be very fast as compared to the FSAM and HSAM methods.
\end{abstract}

